\title{
Cadmium stable isotopes in lunar regolith samples
}

\author{
W. ABOUCHAMI ${ }^{1 *}$, F. WOMBACHER ${ }^{1}, \mathrm{~N}$. \\ BRAUKMÜLLER $^{1}$, S.J.G. GALER ${ }^{2}$ \\ ${ }^{1}$ Institut für Mineralogie and Geologie, Universität zu Köln, \\ Germany (wafa.abouchami@mpic.de) \\ ${ }^{2}$ Max-Planck-Institut für Chemie, Mainz, Germany
}

Mass-dependent stable isotope fractionation of moderately volatile elements in lunar rocks provide contrasting views on the origin, timing and processes that led to the formation of the Moon. While the "giant impact" between the proto-Earth and Theia provides a framework for the greater volatile element depletion of the Moon relative to the Earth, the mechanisms of volatile loss remain unclear [1]. Here, cadmium isotopes provide important constraints on evaporation/condensation processes. In addition, thermal neutron capture ${ }^{113} \mathrm{Cd}(n, \gamma){ }^{114} \mathrm{Cd}$ effects allow estimating the residence time of $\mathrm{Cd}$ in the upper layers of the lunar regolith.

We present TIMS double spike $\mathrm{Cd}$ stable isotope measurements on five Apollo samples, with an up to 20-fold improvement in analytical precision compared to previous data $[2,3,4]$. In order to distinguish silicate-hosted versus vapor-transport related $\mathrm{Cd}$ isotope signals in the regolith, leaching experiments were performed on Apollo 17 orange glass soil 74220 and Apollo 16 mature highlands soil 60500.

Our first results show large stable $\mathrm{Cd}$ isotope fractionation of up to $\sim 5 \% \mathrm{amu}^{-1}$, substantially greater than those of terrestrial and carbonaceous chondrite samples measured alongside the lunar samples. Our data, along those published, demonstrate that $\mathrm{Cd}$ stable isotopes, $\mathrm{Cd}$ abundances and $n$-capture effects correlate with the soil maturity index $(\mathrm{Is} / \mathrm{FeO})$. These trends suggest progressive $\mathrm{Cd}$ depletion with loss of the lighter $\mathrm{Cd}$ isotopes during micrometeorite impacts and efficient impact gardening of the lunar regolith. By contrast, the orange glass soil displays a distinctive light $\mathrm{Cd}$ isotope signature - similar to that of the leachate - most likely acquired from condensation onto glass bead surfaces of a Cd-rich vapor released during volcanic fire fountaining. Additional data will be crucial for refining these initial results regarding the origin and processes that defined the Moon's volatile and isotopic fingerprint and understanding the dynamics occuring at the lunar surface.

[1] Day et al. (2020) EPSL 531, 115998; [2] Sands et al. (2001) EPSL 186, 103-111; [3] Schediwy et al. (2006) EPSL 243, 326-335; [4] Wombacher et al. (2008) GCA 72, 646667. 